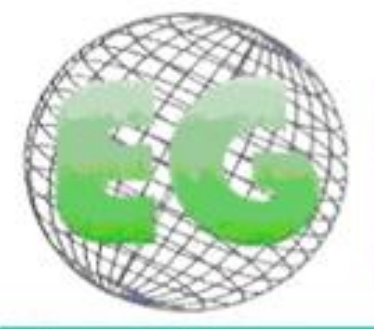

\title{
Experiencias de donación de leche humana en Andalucía-España: un estudio cualitativo
}

Experiences of human milk donation in Andalucía-Spain: a qualitative study

\section{*Machado, Raylane da Silva ${ }^{* *}$ Campos Calderón, Concepción P. ${ }^{* * *}$ Montoya Juárez, Rafael ${ }^{* * * *}$ Schmidt RioValle, Jacqueline}

\begin{abstract}
*Estudiante de Enfermería en intercambio en la Universidad de Granada- España. Becaria del Programa Ciencias sin fronteras del CNPq. Universidade Federal do Piauí. Brasil. E-mail: raylane.s.machado@gmail.com **Facultad de Ciencias de la Salud. Universidad de Granada.
\end{abstract} España.

Palabras Clave: Banco de Leche Materna; Leche Humana; Lactancia Materna; Donación. Keywords: Human Milk Banks; Human Milk; Breastfeeding; Donation

\section{RESUMEN}

Introducción: Los beneficios de la lactancia materna para los bebés han sido bien documentados. Recoger el exceso de leche humana de madres lactantes es un servicio social importante. Los bancos dependen de las donaciones de madres lactantes, por ello la importancia de conocer los aspectos subjetivos que interfieren en la donación.

Objetivo: Conocer las vivencias, creencias, motivaciones y dificultades respecto la donación de leche de un grupo de mujeres donantes en el Banco de Leche Humano de Granada-Andalucia.

Métodos: Estudio descriptivo, con abordaje cualitativo, realizado en el período de mayo y junio de 2013. La muestra estuvo constituida por 7 mujeres que donaron leche en el banco de leche humana del Hospital Virgen de las Nieves de Granada. Para la recogida de datos se utilizaron entrevistas semiestructuradas.

Resultados: Las informantes tenían en su mayoría más de treinta años, con nivel universitario, casadas y con uno o dos hijos como máximo. De los discursos de las madres emergieron las siguientes categorías: Decisión de hacerse donante; Motivaciones donación; Apoyo donación y Dificultades donación.

Conclusión: Como se evidencia, el banco de leche es todavía poco conocido. Todavía existen pocos bancos de leche en España, intentar ampliar esa red sería fundamental para cientos de prematuros que necesitan de esa leche. 


\section{ABSTRACT}

Introduction: The benefits of feeding human milk to infants have been well-documented. Collecting excess human milk from lactating mothers is an important social service. The Human Milk Banks depend on donors, and knowing the subjective aspects of donors seems quite important.

Objective: Compiling the experiences, beliefs, motivations and difficulties concerning milk donation in a group of donors belonging to the Human Milk Bank of Granada- Andalucia.

Methods: A descriptive qualitative study was conducted from May to June of 2013. The sample consisted of 7 women who donated milk to the Human Milk Bank located in the Virgen de las Nieves hospital of Granada. Data was collected using a semi-structured interview.

Results: Most of the interviewees were over thirty years olo d, had completed or were in the process of completing university, married, and had one or two children. During the interview, the following subjects were discussed with the mothers: Decision of becoming a donor, support to the donor, motivation for donating, and difficulties donating.

Conclusion: The results of our study suggest that many mothers are still not aware that there are milk banks present in their location. Expanding the number of milk banks in Spain is essential for hundreds of premature babies who are in need of this necessary resource.

\section{INTRODUCCIÓN}

Está comprobado que la leche materna es el alimento ideal para los recién nacidos. ${ }^{1}$ Es el alimento de elección durante los 6 primeros meses de la vida para todos los niños y se debería prolongar al menos durante todo el primer año y más allá de dicha edad si lo desean tanto la madre como el niño ${ }^{1-3}$.

En situaciones en las que la lactancia materna está contraindicada o difícil (prematuros de bajo peso o casos en que alguna patología de la madre o del neonato impide que él sea amamantado directamente), la Organización Mundial de la Salud ${ }^{1}$, Unicef $^{2}$ y la Asociación Española de Pediatría ${ }^{4}$ (AEP) defienden la leche humana donada como recurso de primera elección para manutención de la lactancia materna. En este contexto los bancos de leche humana (BLH) tienen un papel fundamental para garantizar la oferta de ese alimento.

Un BHL es un dispositivo sanitario que se dedica recoger leche de madres donantes, procesarla, almacenarla y dispensarla, con todas las garantías sanitarias, a los recién nacidos que la puedan necesitar. Para ello, se encarga de la selección de las donantes, así como del almacenamiento, el procesamiento, el análisis y la distribución de la leche ${ }^{2,5}$.

Las donantes son mujeres sanas en el primer semestre de su período de lactancia que tienen secreción de leche superior a las necesidades de su hijo, y que están dispuestas a donarla por libre voluntad ${ }^{6}$. Para ser donante, la madre lactante debe ser sometida a un examen clínico detallado, con el propósito de proteger su salud y la del receptor. Son criterios de exclusión el tabaquismo, consumo de alcohol o sustancias excitantes en cantidades elevadas, prácticas de riesgo de enfermedades transmisibles, enfermedades crónicas o consumo actual de algún tipo de medicamento. ${ }^{7}$ 
El primer banco de leche del mundo se creó en Viena en el año 1909. Desde entonces los bancos de leche fueron implantándose en numerosos países. Existen asociaciones nacionales sólidamente establecidas en Estados Unidos, Reino Unido, Italia y $\mathrm{Brasi}^{5,7,8}$. Este último lidera mundialmente las redes nacionales de $\mathrm{BLH}^{8,9,10}$, y cuenta con la mayor (213 bancos, en 2013) ${ }^{10}$ y más compleja red, constituida como estrategia del Ministerio de Salud para mejorar la salud de las madres y los lactantes ${ }^{5,7,11}$.

En el año 2008 se creó la Asociación Española de Bancos de Leche. Uno de sus principales objetivos es promover la creación de bancos de leche en España y facilitar la cooperación entre bancos de leche nacionales y otros bancos de leche europeos. ${ }^{5,7}$ Desde la apertura del primer BLH en 2001, España ya cuenta con siete bancos de leche, cuyo fin es alimentar a cientos de neonatos ${ }^{12}$. El Banco de Leche del Hospital Virgen de las Nieves de Granada fue instalado basado en el modelo brasileño y comenzó su funcionamiento en el mes de Mayo de 2010 siendo único en funcionamiento en toda Andalucía.

Este estudio tiene como objetivo conocer las vivencias, creencias, motivaciones y dificultades a respecto de donación de leche de un grupo de mujeres que donan leche en Granada. Conocer esos significados puede reorientar las prácticas llevadas a cabo por los profesionales involucrados con el binomio la madre-bebé, para que exista la sensibilización de más madres y el consiguiente aumento del número de donantes.

\section{MÉTODO}

Se trata de un estudio descriptivo, desarrollado según los principios de la investigación cualitativa, que tiene como característica ofrecer al investigador la posibilidad de captar la manera por la cual los individuos piensan y reaccionan, permitiendo conocer la dinámica y la estructura de la situación bajo el punto de vista de quien la vive ${ }^{13}$. En este sentido, el enfoque de este estudio fue fenomenológico, siendo el fenómeno a estudiar la donación de leche a los BLH

El proyecto fue aprobado por el Comité de Ética de la Investigación Biomédica de la Provincia de Granada. La población estuvo constituida de 7 mujeres que donaron leche en el período de mayo y junio de 2013 en el BLH del Hospital Virgen de las Nieves de Granada. Después de que aceptaran y firmaran el consentimiento informado, fueron realizadas entrevistas semi-estructuradas en un único encuentro con cada informante. En las entrevistas constaban como preguntas de orientación: ¿Por qué se decidió a ser donante de leche?, ¿Qué le ha parecido a su familia y amigos esta decisión?, ¿Ha influido el personal sanitario (enfermería y médicos) en su decisión de donar?, ¿Tuvo alguna dificultad para donar?

Para el análisis e interpretación de los datos, fue seguido un modelo estructurado adaptado de Flick $^{14}:$ 1) transcripción de las entrevistas, 2) descubrimiento de códigos y elaboración de familias o categorías de análisis; 3 ) codificación de las entrevistas en base a las categorías identificadas; 4) interpretación de los datos en el contexto en el que fueron recogidos.

Se empleó como herramienta informática el programa Atlas-ti 6.2; cuyo objetivo es facilitar el análisis cualitativo al proporcionar fiabilidad, rigor y calidad a los resultados ${ }^{13}$. 


\section{RESULTADOS}

La tabla I presenta las características sociodemográficas de las 7 mujeres que participaron del estudio.

Tabla I. Caracterización de las madres donantes del banco de leche de Granada,2013

\begin{tabular}{cccccc}
\hline INFORMANTES & $\begin{array}{c}\text { EDAD } \\
\text { MADRE }\end{array}$ & ESTADO CIVIL & HIJOS & OCUPACION & ESCOLARIDAD \\
\hline M1 & 39 & Soltera & 1 & Bombera & $\begin{array}{c}\text { Formación } \\
\text { Profesional }\end{array}$ \\
\hline M2 & 34 & Casada & 2 & Matemática & Universitaria \\
\hline M3 & 21 & Casada & 1 & Estudiante & $\begin{array}{c}\text { Formación } \\
\text { Profesional }\end{array}$ \\
\hline M5 & 32 & Casada & 1 & Doctora en letras & Universitaria \\
\hline M6 & 35 & Casada & 2 & Enfermera & Universitaria \\
\hline M7 & 27 & Casada & 1 & Médica & Universitaria \\
\hline
\end{tabular}

De acuerdo a las categorías de análisis, se han encontrado los siguientes resultados:

\section{- Decisión de hacerse donante}

De los factores que influyeron en la decisión de hacerse donante de leche humana emergió dos subcategorías: Información Banco de Leche y Percepción entorno sociofamiliar (tablall).

-Información Banco de Leche: trata de cómo las mujeres llegan a conocer el banco de leche o como supieron de la posibilidad de donación de leche humana. Ellas conocen la existencia del banco de leche, en un primer momento, a través de los folletos que se les entregan tras el alta hospitalaria post-parto. Posteriormente, se les despierta la curiosidad sobre el tema y realizan una búsqueda personal de información sobre cómo pueden ellas hacerse donantes.

- Percepción entorno socio-familiar: hace referencia a la opinión que tiene la familia y los amigos sobre la decisión de ser donante de leche humana. Las donantes refieren que su entorno más cercano (su familia y amigos) suelen tener una reacción muy positiva al enterarse de que ellas contribuyen con su leche, ya que muchos de ellos, no conocen la existencia del banco de leche ni su finalidad. 
Tabla II. Pasajes entrevista a cerca de la decisión de hacerse donante

\begin{tabular}{|c|c|}
\hline SUBCATECORIAS & PASAJE ENTREVISTA \\
\hline \multirow{3}{*}{$\begin{array}{l}\text { Información } \\
\text { Banco de Leche }\end{array}$} & $\begin{array}{l}\text { Cuando te vas de alta en el hospital(tras el parto) te } \\
\text { dan entre todos los papeles de la niña te dan un } \\
\text { papel donde te hablan de la donación de leche y } \\
\text { luego además una amiga mía que es pediatra pues } \\
\text { fue la que lo comentó personalmente (M6) }\end{array}$ \\
\hline & $\begin{array}{l}\text { Me enteré de la donación de leche por internet, } \\
\text { concretamente por una asociación que se llama } \\
\text { Mami Lactancia y me comentaron que se } \\
\text { necesitaba leche (M2). }\end{array}$ \\
\hline & $\begin{array}{l}\text { cuando mi hija estaba ingresada en el hospital } \\
\text { porque se puso mala, leí los papeles (sobre el } \\
\text { banco de leche) y fui a informarme(M3). }\end{array}$ \\
\hline \multirow[b]{2}{*}{$\begin{array}{l}\text { Percepción } \\
\text { entorno socio- } \\
\text { familiar }\end{array}$} & $\begin{array}{l}\text { De hecho todo el mundo alucina porque nadie sabe } \\
\text { que hay un banco de leche.(M1). }\end{array}$ \\
\hline & $\begin{array}{l}\text { Me ven con el saca leche y se lo comenté, y le } \\
\text { pareció a todos maravilloso. Me dicen que soy muy } \\
\text { buena persona por eso, me da vergüenza pero yo } \\
\text { no lo hago por ser buena persona pero la verdad es } \\
\text { que tienen una reacción muy positiva(M4-P5) }\end{array}$ \\
\hline
\end{tabular}

\section{- Motivaciones donación}

Cuando analizamos los discursos de las madres acerca de las motivaciones que les Ilevaron a hacerse donantes de leche, emergieron cuatro subcategorías (tabla III).

- Creencias de los beneficios de la lactancia: las mujeres participantes atribuyen una serie de significados positivos a la lactancia materna. Las madres creen que la leche materna es lo mejor que se puede dar a los niños, basándose en su propia experiencia como madres.

- "Tener mucha leche": las madres expresaron que una de las principales motivaciones para donar leche fue la producción excesiva, lo cual les provocaba ingurgitación mamaria, con el consecuente dolor y molestias. La donación se convierte entonces, en una oportunidad para aliviar los síntomas de la ingurgitación.

- Altruismo: otra de las motivaciones de las madres donantes era la satisfacción de actuar desinteresadamente en beneficio de otro, en ese caso, de bebés que se encuentran en condiciones difíciles, pues la mayoría de los niños receptores son prematuros.

- "Por si le pasa a mi bebé": además del altruismo algunas madres manifiestan que se deciden a donar porque piensan que, en el caso de que un futuro hijo suyo lo necesitara, sería muy bueno que alguien donara leche para él también. 
Tabla III. Pasajes de la entrevista a cerca de: motivaciones donación

\begin{tabular}{|c|c|}
\hline SUBCATEGORIAS & PASAJE ENTREVISTA \\
\hline \multirow{2}{*}{$\begin{array}{l}\text { Creencias de los } \\
\text { beneficios de la } \\
\text { lactancia }\end{array}$} & $\begin{array}{l}\text { A mis dos hijos los he criado con lactancia materna } \\
\text { exclusiva hasta los seis meses sin ningún biberón, ni } \\
\text { manzanilla, ni agua, ni ningún tipo de complemento } \\
\text { y han sido unos niños que se han criado muy sanos } \\
\text { y muy gorditos y muy grandes. Entonces yo pensaba } \\
\text { que si yo pudiera dar mi leche a otros niños, ¿no?, } \\
\text { porque esta leche es muy buena, entonces me } \\
\text { decidi que quería donar leche, eso fue (M7) }\end{array}$ \\
\hline & $\begin{array}{l}\text { como estoy muy concienciada de la lactancia } \\
\text { materna y he tenido la suerte de que estoy muy } \\
\text { informada y que he tenido mucho apoyo en mi } \\
\text { entorno y en mi familia pues me decidi a ser } \\
\text { donante, porque creo es necesario para los niños. } \\
\text { (M5) }\end{array}$ \\
\hline \multirow{3}{*}{$\begin{array}{c}\text { Tener mucha } \\
\text { leche }\end{array}$} & $\begin{array}{l}\text { Porque tenía muchísima leche. Entonces me } \\
\text { comentaron lo del banco de leche y me decidi } \\
\text { hacerme donante de leche; me daba pena tirarta" } \\
\text { (M2) }\end{array}$ \\
\hline & $\begin{array}{l}\text { Tenía mucha cantidad y como me sobraba, pues } \\
\text { antes de tírala pues dársela a los bebés que la } \\
\text { necesitan. (M3) }\end{array}$ \\
\hline & $\begin{array}{l}\text { Tenía leche de sobra porque la niña solamente } \\
\text { tomaba un pecho y el otro no le hacía falta. No } \\
\text { quería más pecho y la leche duele cuando no se } \\
\text { saca. Entonces, me lo habian comentado en el } \\
\text { hospital y, pues mira, para tirar la leche que es una } \\
\text { pena, pues se la doy.(M6) }\end{array}$ \\
\hline
\end{tabular}

\begin{tabular}{|c|c|}
\hline \multirow{2}{*}{ Altruismo } & $\begin{array}{l}\text { Pensé que (donar leche) era bueno para los niños } \\
\text { que no podian tener leche materna y que además } \\
\text { eran prematuros. (M4) }\end{array}$ \\
\hline & $\begin{array}{l}\text { Si hay una forma de ayudar a otra persona, y } \\
\text { encima son niños, que son lo más bonito del mundo, } \\
\text { pues ahi estoy yo. (M5) }\end{array}$ \\
\hline \multirow{2}{*}{$\begin{array}{c}\text { Por si le pasa a mi } \\
\text { bebé }\end{array}$} & $\begin{array}{l}\text { Puede pasarme a mi (no tener leche) con el } \\
\text { siguiente hijo, entonces pues mira... (M1) }\end{array}$ \\
\hline & $\begin{array}{l}\text { En el día de mañana se tengo otro hijo y yo no } \\
\text { pudiera darle (el pecho) por lo que fuera, pues me } \\
\text { gustaría que le ayudaran. (M3) }\end{array}$ \\
\hline
\end{tabular}




\section{- Apoyo donación}

El entorno de las madres, influye de manera positiva en la disposición de las participantes en continuar con la donación (tabla IV). En este sentido, hemos encontrado los siguientes elementos de apoyo:

- Apoyo Banco de Leche: apoyo que reciben las madres desde del personal del Banco de leche para seguir siendo donantes. Las donantes relatan que se sienten reforzadas por los profesionales sanitarios. Además, atribuyen una gran importancia al esfuerzo constante que hacen para mantenerlas motivadas.

- Apoyo familiar donación: las mujeres donantes reciben también apoyo por parte de su familia, sobre todo de su pareja. En la mayoría de los casos, éste se concretaba en trasladarlas a ellas al banco de leche, o llevar la leche ellos mismos.

Tabla IV. Pasajes de la entrevista a cerca de: apoyo donación

\begin{tabular}{|c|c|}
\hline SUBCATEGORIAS & PASAJE ENTREVISTA \\
\hline \multirow[b]{2}{*}{$\begin{array}{c}\text { Apoyo Banco de } \\
\text { Leche }\end{array}$} & $\begin{array}{l}\text { Algunas veces me desmotivo, y cuando voy al } \\
\text { banco de leche y veo con la alegría que me reciben } \\
\text { y agradecen, pues sí que me motiva bastante para } \\
\text { continuar (M5) }\end{array}$ \\
\hline & $\begin{array}{l}\text { La verdad es que es muy majo el personal del } \\
\text { hospital, del banco de leche es muy muy majo. } \\
\text { Cuando voy y todo mundo nos dice: "que bien, que } \\
\text { bien viene y que alegría", que eso es muy } \\
\text { beneficioso para los niños, pues que te vuelve a } \\
\text { motivar. (M2) }\end{array}$ \\
\hline \multirow{2}{*}{$\begin{array}{l}\text { Apoyo familiar } \\
\text { donación }\end{array}$} & $\begin{array}{l}\text { Mi marido sí que me echa una mano de vez en } \\
\text { cuando, llevando la leche. Pues con un bebé ya } \\
\text { tengo mucho lio, y a mi el hospital me pilla lejos de } \\
\text { casa (M2). }\end{array}$ \\
\hline & $\begin{array}{l}\text { Mi familia está de acuerdo en que done leche y } \\
\text { cuándo pueden, me llevan a Granada para que } \\
\text { lleve la leche (M3) }\end{array}$ \\
\hline
\end{tabular}

\section{- Dificultades donación}

Esta categoría describe los obstáculos que las madres perciben para comenzar y mantenerse como donantes de leche. Las participantes identifican cuatro barreras principales (tabla V):

- Desconocimiento del resto de los profesionales sanitarios: la falta de información acerca de la existencia de banco de leche por parte de los profesionales sanitarios que atienden a las mujeres en otras unidades (hospital, centro de salud), condicionó que muchas mujeres tuvieran que esforzarse doblemente, primero para localizar el banco de leche y, después, para hacerse donante. 
- Distancia con respecto al banco de leche: uno de los factores que más dificultan la donación de leche es la distancia que, en su mayoría, tienen que recorrer desde sus domicilios en el interior de la provincia para llevar la leche al centro, en el BLH.

- Incomprensión en el trabajo: gran parte de las madres refieren que no reciben apoyo alguno en su trabajo, antes al contrario incluso, reciben insinuaciones sobre si deberían de dejar la lactancia materna o, por lo menos, introducir de manera temprana la alimentación complementaria, con el fin de que pudieran rendir más en el trabajo sin tener que salir para amamantar.

- Disminución de la leche por el propio proceso de lactancia materna: las mujeres también destacan el hecho de que, con el tiempo, ya no producen tanta leche, como en el momento en el que se decidieron a ser donantes, de manera que empiezan a disminuir la frecuencia en las donaciones. Aunque, en este caso se trata de un proceso natural, las mujeres lo experimentan como una dificultad más para continuar siendo donantes.

Tabla V. Pasajes de la entrevista a cerca de: dificultades donación

\begin{tabular}{|c|c|}
\hline SUBCATEGORIAS & PASAJE ENTREVISTA \\
\hline \multirow{2}{*}{$\begin{array}{l}\text { Desconocimiento } \\
\text { del resto de los } \\
\text { profesionales } \\
\text { sanitarios }\end{array}$} & $\begin{array}{l}\text { Con respecto a la donación tampoco nadie me había } \\
\text { contado nada. De hecho, ni mi médico sabía de } \\
\text { donación de leche, ni en el centro de salud tampoco. } \\
\text { (M2) }\end{array}$ \\
\hline & $\begin{array}{l}\text { Como yo pregunté en el hospital y en un principio } \\
\text { nadie supo decime si habia o no habia, (banco de } \\
\text { leche) fue también una cosa personal(donar } \\
\text { leche).(M1) }\end{array}$ \\
\hline \multirow[t]{2}{*}{$\begin{array}{l}\text { Distancia con } \\
\text { respecto al banco } \\
\text { de leche }\end{array}$} & $\begin{array}{l}\text { Para llegar en el banco de leche de Granada tardo } \\
\text { casi una hora en ir y otra en venir. Entonces, } \\
\text { muchas veces, o llevaba el niño conmigo para no } \\
\text { dejarlo mucho rato solo, y hombre, un viaje así para } \\
\text { llevar la leche, pues yo que sé, me daba lástima. } \\
\text { Porque, muchas veces, volviendo para casa, se } \\
\text { ponía un poco desesperado. Luego ir sola tampoco } \\
\text { me gusta, porque claro, si no me lo llevo, no puedo } \\
\text { estar con él [...JCreo que deberían de implantarse } \\
\text { bancos de leche en más provincias." (M5) }\end{array}$ \\
\hline & $\begin{array}{l}\text { De hecho, me cuesta trabajo. No tengo forma de } \\
\text { llevarla (la leche), porque además me pilla bastante } \\
\text { lejos. Entonces, como que me desanima un poco. } \\
\text { Tengo que esperar hasta tener cita en el medico, } \\
\text { porque no pueden venir a recogerla." (M3) }\end{array}$ \\
\hline \multirow{2}{*}{$\begin{array}{c}\text { Incomprensión en } \\
\text { el trabajo }\end{array}$} & $\begin{array}{l}\text { Incluso para la lactancia matema no hay un apoyo } \\
\text { en el trabajo. Por la experiencia que tuve con mi otra } \\
\text { hija, no hay ningún apoyo especial, de hecho, nadie } \\
\text { te anima... mucho menos para la donación. (M7) }\end{array}$ \\
\hline & $\begin{array}{l}\text { Me han insinuado (en el trabajo) que yo no tenía que } \\
\text { estar dando el pecho al niño o que tenía que está } \\
\text { dando otra comida más temprano, para que el niño } \\
\text { pudiera aguantarse (mientras estoy trabajando (M4) }\end{array}$ \\
\hline \multirow{2}{*}{$\begin{array}{l}\text { Disminución de la } \\
\text { leche por el } \\
\text { propio proceso de } \\
\text { lactancia materna }\end{array}$} & $\begin{array}{l}\text { Llevo tres semanas que tengo más dificultad para } \\
\text { sacame leche porque ... pues se ve que me he } \\
\text { regulado un poquito más con el bebé. Ya no se me } \\
\text { hincha tanto (el pecho) y me cuesta trabajo sacame } \\
\text { leche. (M7) }\end{array}$ \\
\hline & $\begin{array}{l}\text { Ahora mismo que la niña ya está más grande y toma } \\
\text { mejor la leche, ya hace ya tiempo que yo que no doy } \\
\text { leche (M6) }\end{array}$ \\
\hline
\end{tabular}




\section{DISCUSIÓN}

Los resultados de este estudio presentan un acercamiento al tema de la donación de leche humana en Andalucía, aportando la perspectiva de las donantes y la dinámica con la cual se desarrolla esta práctica en España.

Las informantes tenían en su mayoría más de treinta años, con nivel universitario, casadas y con uno o dos hijos como máximo, esos datos son similares a los que maneja el Hospital 12 de Octubre (el mayor banco de leche de toda España) los cuales indican que las madres donantes suelen tener unos 30 años, donan con su primer hijo, tienen pareja estable y más de la mitad son universitarias ${ }^{6}$.

A pesar de la importancia de recoger la leche humana en BLH para la supervivencia de los recién nacidos, la divulgación sobre el tema todavía está muy limitada. La información se proporciona principalmente en el proceso de hospitalización para el parto en la maternidad, no cubriendo la red de asistencia prenatal o de centros de salud (tabla 2). Ese mismo factor se puede observar en otros estudios ${ }^{14,15}$ que apuntan la desinformación como uno de los principales problemas para que las madres no se decidan a la donación de leche.

Cabe la hipótesis de que muchas donantes potenciales ya han frustrado sus intenciones, no llegando a poner en práctica su papel de donante. Nuestros datos señalan que, aunque tengan conocimiento de la existencia del BLH a través de asociaciones de apoyo a lactancia materna o a través de internet, es el deseo personal el principal factor para ser donantes.

Es pertinente sugerir que sean replanteadas las rutinas de recolección de la leche, con la implantación de recogida de la leche en el propio domicilio de las donantes, salvando de esta forma, una de las mayores dificultades apuntadas por las donantes: la distancia que tienen que recorrer para llevar la leche donada. Este sistema ya funciona en Brasil $^{16}$ y ha logrado mucho éxito ampliando el número de donantes así como la frecuencia de donación.

Actualmente, solamente hay un banco de leche en Andalucía, que tiene sede en Granada. Las propias participantes abogan por la implantación de más unidades de BLH. Esta medida facilitaría un acercamiento con la población donante y aumentaría la cobertura a los niños que necesiten leche donada.

Un aspecto importante de nuestros resultados se refiere al reconocimiento de las donantes en relación a las ventajas y cualidad nutricional de la leche materna. El altruismo surge como motivación principal para nuestras participantes, y reconocen que la decisión de donar, atañe exclusivamente a la mujer. El segundo motivo más citado es el exceso de producción láctea, cuando identifican que podrían aprovechar el exceso de leche evitando la pérdida del mismo.

Otra cuestión reseñada por las donantes, y que les hace mostrarse más sensibles hacia la donación de leche, es ponerse en el lugar de las madres que pasan por dificultades de amamantar a sus hijos, e incluso el hecho de pensar que esto les podría ocurrir a ellas en un futuro.

En otros estudios realizados en Brasil ${ }^{14,15,16}$ y en Guatemala ${ }^{8}$ se destacan las siguientes razones y sentimientos involucrados en la donación de la leche: la 
solidaridad, el amor, la comodidad, el valor de la leche, la satisfacción personal, la retribución y ayudar a los demás. En los estudios de Francia ${ }^{17}$ y EEUU ${ }^{18}$ el motivo que llevó a las madres a donar su leche fue la turgencia mamaria siendo orientadas por profesionales de salud.

Es imprescindible decir que el apoyo ofrecido por el BLH así como el apoyo de la familia ${ }^{18}$ son esenciales para la manutención de esas mujeres como donantes tal y como ellas mismas citan (tabla 4). Una barrera importante a la donación es la falta de apoyo para la lactancia en el trabajo y por consiguiente para la donación, así como la reducción de la producción láctea que inicialmente les motivó a donasen.

\section{CONCLUSIONES}

Como se evidencia, el banco de leche es todavía poco conocido, debido principalmente, a la falta de divulgación del mismo. Ampliar la red de bancos de leche en España sería fundamental para cientos de prematuros que necesitan de esa leche, y no pueden acceder a los mismos debido a la distancia que existe desde sus domicilios.

Se demuestra la necesidad de una mayor promoción y orientación en materia de donación de leche humana, haciendo especial hincapié en las razones que aducen las mujeres de este estudio (altruismo, beneficios de la lactancia, "Tener mucha leche", ponerse en el lugar de otras madres), así como promover el apoyo de las familias y del personal sanitario.

Las conclusiones que se pueden extraer de este estudio están limitadas por el uso de la muestra de conveniencia de donantes, no se sabe cómo de representativas sus opiniones y experiencias son de la población total de donantes de leche.

Para incentivar la captación de nuevas donantes de leche humana se deberían realizar acciones educativas/formativas en los servicios de atención de prenatal, maternidad, guarderías y reforzar el sistema de comunicación con otros sectores sanitarios.

Por esto, también debería invertirse en aumentar la investigación y la producción científica con el fin de mejorar y garantizar la calidad de vida de los niños cuyas madres no pueden amamantar.

\section{BIBLIOGRAFÍA}

1. World Health Organization. Infant and young child nutrition: global strategy on infant and young child feeding. Geneva: World Health Organization; 2002.

2. García-Lara N R, García-Algar O, Pállas-Alonso C R. Sobre bancos de leche humana y lactancia materna. An Pediatr (Barc). 2012;76(5):247-249. Disponible en: URL: http://zl.elsevier.es/es/revista/anales-pediatria-37/sobre-bancos-leche-humanalactancia-materna-90132447-editorial-2012?bd=1

3. WHO/UNICEF. Protecting, promoting and supporting breastfeeding: the special role of maternity services. Geneva: World Health Organization ;1990.

4. Hernández-Aguilar MT, Aguayo-Maldonado J. La lactancia materna. Cómo promover y apoyar la lactancia materna en la práctica pediátrica. Recomendaciones del Comité de Lactancia de la Asociación Española de Pediatría. An. Pediatr (Barc). 
2005;63(4):340-56. Disponible en: URL: http://zl.elsevier.es/es/revista/analespediatria-37/articulo/la-lactancia-materna-como-promover-13079817

5. Gormaz M, Roqués V, Dalmau J, Vento M, Torres E, Vitoria I. Actividad de un banco de leche humana implantado en una unidad neonatal. Acta Pediatr Esp 2011; 69 (6): 283-287.

6. Sierra Colomina $\mathrm{G}$, et al. Características de las mujeres donantes de un banco de leche materna y relación con el tiempo de donación. An Pediatr (Barc). 2013.Disponible en: URL: http://dx.doi.org/10.1016/j.anpedi.2013.05.017

7. Vázquez-Román S, Alonso-Díaz C, Medina-López C, Bustos-Lozano S, Martínez- Hidalgo MV, Pallás- Alonso CR. Puesta en marcha del banco de leche materna donada en una unidad neonatal. An Pediatr (Barc) 2009; 71 (4): 343-348.

8. De León Soto J F, Arreaga Fion G L. Conocimientos, actitudes y percepciones de madres Lactantes respecto a la donación de leche materna al Banco de leche humana del Hospital Nacional Pedro de Bethancourt [tesis doctoral]. Guatemala: Universidad de San Carlos de Guatemala; 2008

9. Da Silva SL. Alegações maternas para doação de leite [tesis doctoral]. Recife: Universidade Federal de Pernambuco; 2010.

10. Brasil. Ministerio da Saúde. A Rede Brasileira de Bancos de Leite Humano : Bancos de Leite Humano - Localização e Relatórios. Disponible en: http://producao.redeblh.icict.fiocruz.br/portal blh/blh brasil.php

11. Da Silva Machado R, Soares LS, Da Silva Freitas GR. Red brasileña de bancos de leche humana: revisión de los aspectos históricos y políticos. Nutr. Hosp. [revista en la Internet] 2012;(Supl. 3)27:1-87 [citado 2013 Sep 06].

12. Asociación Española de Bancos de leche Humana. Tu banco de leche. Disponible en: http://www.aeblh.org/tu-banco/

13. Flick, U. Introdução à pesquisa qualitativa. 3ee. Porto Alegre: Artmed; 2009.

14. Galvão M T G, Vasconcelos S G, Paiva S S. Reasons Women donate breast milk. Acta paul. enferm. [serial on the Internet]. 2006 June [cited 2013 Sep 06] ; 19( 2 ): 157-161. Available from: http://www.scielo.br/scielo.phb?script=sci arttext\&pid=S0103-

21002006000200006\&lng=en.http://dx.doi.org/10.1590/S0103-21002006000200006 .

15. Dias RC, Baptista IC, Gazola S, Rona MSS, Matioli G. Human milk bank donors' profile at Hospital Universitário de Maringá, State of Paraná, Brazil. Acta Sci Health Sci. 2006;28(2):153-8.

16. Tomaz ACP, Loureiro LVM, Oliveira TS, Montenegro NCMF, Junior EDA, Soriano CFR, Cavalcante JC. The human milk donation experience: motives, influencing factors and regular donation. Journal of Human Lactation.2008; 24(1):6976.

17. Azema E, Callahan S. Breast Milk in France: A Portrait of tropical donor and the utility of milk banking in the French breastfeeding context. Journal of Human lactation. 2003; 19(2): 199-202.

18. Osbaldiston R, Mingle LA. Characterization of Human Milk Donors. Journal of human lactation. 2007; 23(4):350-7

Recibido: 09 de febrero de 2014; Aceptado: 04 de abril de 2014

ISSN 1695-6141

๑) COPYRIGHT Servicio de Publicaciones - Universidad de Murcia 